\title{
Electrochemical Anionic Polymerization of 4-Vinylpyridine in Pyridine
}

\author{
S. N. BHADANI and G. PARRAVANO, Department of Chemical and \\ Metallurgical Engineering and Macromolecular Research Center University \\ of Michigan, Ann Arbor, Michigan 48104
}

\section{Synopsis}

The preparation of poly-4-vinylpyridine (poly-4VP) by electrochemical polymerization of 4-vinylpyridine (4VP) in pyridine containing sodium tetraphenylboron $\left(\mathrm{NaBPh}_{4}\right)$ is described. Information on the influence of monomer concentration, current density, polymerization rate, molecular weight, and electrochemical efficiency is presented. The polymerizations were performed under conditions of constant electrolysis current. Polymer formed in the cathodic compartment only, where a red-orange solution developed after about $15 \mathrm{~min}$ of electrolysis time. The optical absorption spectra of these colored solutions were studied. Cyclic voltammograms of $4 \mathrm{VP}$ in pyridine and $\mathrm{NaBPh}_{4}$ are also reported, and the influence of the scan rate upon peak current is described. The results indicate that the polymerization was anionic and nonterminating. The characteristics of the electrochemical polymerization of $4 \mathrm{VP}$ in pyridine are compared with those of the same monomer in liquid $\mathrm{NH}_{3}$. In the former case, the catholyte was homogeneous, and polymer growth occurred in the liquid phase, while in the latter growth took place in a heterogeneous environment. Kinetic consequences of these physical differences are pointed out. Suggestions for the mechanism of this electrochemical initiation are advanced.

\section{INTRODUCTION}

In a recent study of the electrochemical polymerization of 4-vinylpyridine (4VP) in liquid $\mathrm{NH}_{3}$ containing $\mathrm{NaCl}$ or $\mathrm{NaN}_{3}$, it was shown that a polymeric red-orange mass formed exclusively around the cell cathode (Pt). This system produced high electrochemical yields and polymers of high molecular weight $\left(\sim 10^{5}\right)$. Chain transfer or termination with monomer or solvent did not occur readily. However, the originally homogeneous liquid phase soon became heterogeneous because of the insolubility of poly$4 \mathrm{VP}$ in liquid $\mathrm{NH}_{3}$.

In the present communication we report investigations of the electrochemical anionic polymerization of $4 \mathrm{VP}$ in pyridine. Pyridine represents an attractive medium for electrochemical polymerizations since it dissolves a variety of monomers and polymers including poly-4VP, it provides a good electrical conducting medium by dissolving a large number of inorganic and organic salts, and, since it has $\mathrm{p} K_{b}=8.8$, it may be expected 
to support many anionic as well as free radical polymerizations. In this latter instance, it has already been tested in a polymerization system with chemical initiation. ${ }^{2}$ Pyridine as a solvent for electrochemical polymerization has not been fully tested, since only a short report on the electrochemical polymerization of styrene in pyridine has appeared. ${ }^{3}$ For these reasons it seemed interesting to study whether 4VP could be polymerized electrochemically in pyridine and, if polymerization resulted, to assess some of the characteristics of the polymerization and to verify whether this polymerization possessed features different from the known heterogeneous, electrochemical, living polymerization of this monomer in liquid $\mathrm{NH}_{3}$.

The results of this study are reported in this communication together with information on monomer reduction potentials obtained by cyclic voltammetry under conditions similar to those employed for polymerization.

\section{EXPERIMENTAL}

\section{Materials}

Pyridine was dried over $\mathrm{CaH}_{2}$ for several days at room temperature and then fractionally distilled in a wetted-wall column under $\mathrm{N}_{2}$ atmosphere. The middle fraction was collected in a flask containing $\mathrm{CaH}_{2}$. The flask was attached to a vacuum system and the pyridine was flash distilled into a thoroughly dried and evacuated flask with $\mathrm{CaH}_{2}$. The final purified pyridine was kept stirred over $\mathrm{CaH}_{2}$ on the vacuum manifold. 4VP was purified and stored as described previously. ${ }^{4}$ Sodium tetraphenyl-boron $\left(\mathrm{NaBPh}_{4}\right)$ from a commercial source was used without further purification.

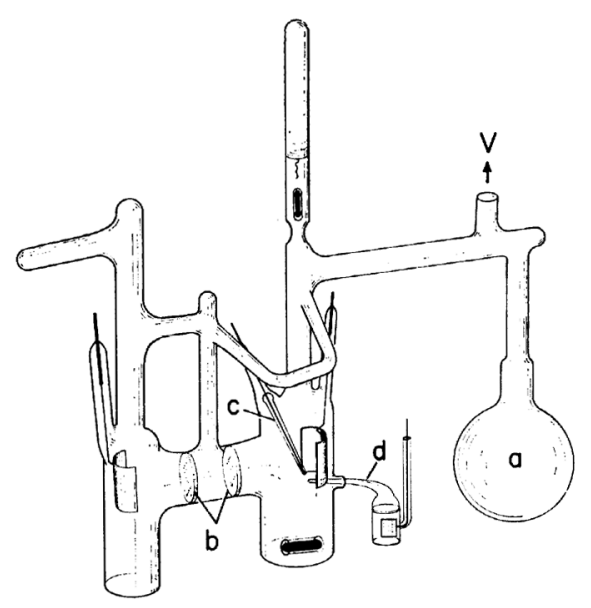

Fig. 1. Electrolytic cell used to study polymerization of 4VP in pyridine: $(a)$ filling bulb; (b) fritted glass disks; (c) micro Pt electrode; $(d)$ reference electrode; $(v)$ vacuum line. 


\section{Equipment}

The electrolytic cell used is shown in Figure 1. Two Pt electrodes $(1 \times$ 1 in.) were housed in the cell which was divided by two fritted glass dises (medium porosity) of $2 \mathrm{~cm}$ diameter. The cathode compartment of the electrolytic cell contained also a planar $\mathrm{Pt}$ microelectrode ( $1 / 6$ in. diameter). $\mathrm{An} \mathrm{Ag} / \mathrm{AgCl}$ reference electrode with a Luggin capillary was also sealed to the cathode compartment. The preparation of the $\mathrm{Ag} / \mathrm{AgCl}$ reference electrode has already been described. ${ }^{5}$ This type of multielectrode electrolytic cell could be used for electrochemical polymerization and analytical work under high vacuum conditions. A Wenking 6IRH potentiostat, coupled with a Wavetek Model 111 voltage function generator and a Honeywell $x-y$ recorder were employed.

\section{Polymerization Procedure}

A known amount of $\mathrm{NaBPh}_{4}$ was introduced into the filling bulb ( $d$ in Fig. 1), and the electrolytic cell was attached to the vacuum line. The apparatus was evacuated and electrically baked at $\sim 10^{-6}$ torr and $60^{\circ} \mathrm{C}$ for 12-16 hr. The vacuum manifold and connected parts were flushed several times with purified $\mathrm{N}_{2}$. The required amount of pyridine was flash distilled into the bulb and degassed thoroughly by repeated freezing and thawing cycles. The apparatus was sealed off from the vacuum line and the contents of the filling bulb were thoroughly mixed at room temperature, and transferred to the anode, cathode, and reference electrode compartments. The monomer was introduced into the cathode compartment by breaking the ampule attached to the cathode compartment. The total volume of the solution was $105 \mathrm{ml}$ and that of the catholyte $65 \mathrm{ml}$. All electrolytic runs were carried out at room temperature. At the end of a run the electrolyzed monomer solution containing dissolved polymer was slowly added to well stirred tetrahydrofuran (THF) at $0^{\circ} \mathrm{C}$. The polymer precipitated as white flock; it was filtered, washed with THF; and vacuum-dried overnight at $50^{\circ} \mathrm{C}$. Polymer molecular weights were obtained as described previously. ${ }^{1}$

\section{RESULTS}

\section{Cyclic Voltammetry}

The cyclic voltammograms of $4 \mathrm{VP}$ were obtained in anhydrous pyridine solution containing $3 \times 10^{-2}$ mole/l. $\mathrm{NaBPh}_{4}$. The monomer exhibited a cathodic peak at $-2.15 \mathrm{~V}$ (Fig. 2). At higher potential $(>-2.4 \mathrm{~V}$ ) a new peak appeared, probably from the reduction of pyridine or $\mathrm{NaBPh}_{4}$. An anodic peak was found at $-0.6 \mathrm{~V}$. It was attributed to the oxidation of the reduction product formed at potential $>-2.25 \mathrm{~V}$, since it did not appear when the potential scan was limited to $-2.25 \mathrm{~V}$. The anodic peak at $-0.6 \mathrm{~V}$ occurred also in the absence of $4 \mathrm{VP}$. 


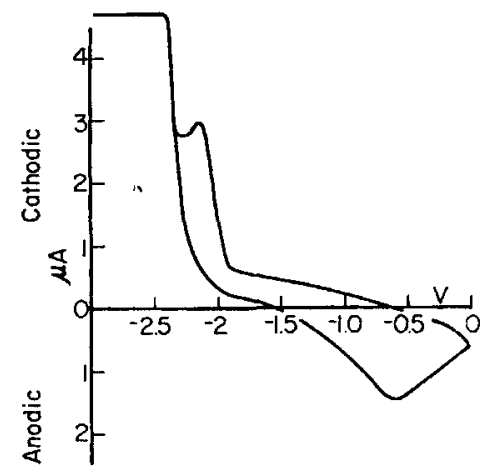

Fig. 2. Cyclic voltammogram of $4 \mathrm{VP}, 8.1 \mathrm{~mole} / \mathrm{l}$. in pyridine with $30 \mathrm{mmole} / \mathrm{l}$. $\mathrm{NaBPh}$ at $25^{\circ} \mathrm{C}$. Planar Pt microelectrode.

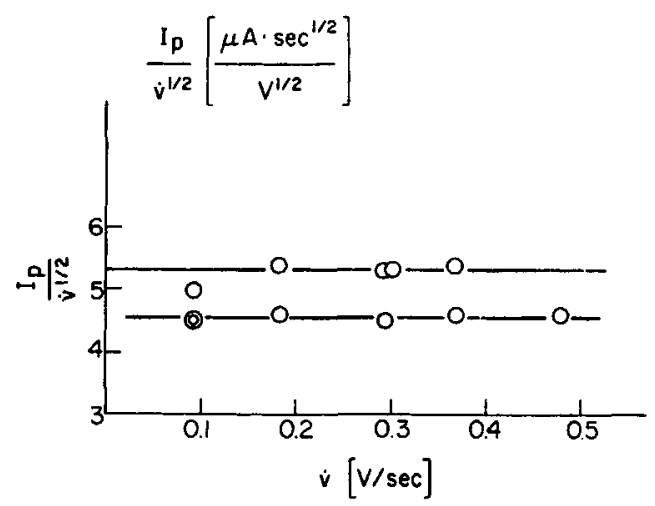

Fig. 3. Plot of $I_{\mathrm{p}} / v^{1 / 2}$ vs. $\dot{v}$ for the cathodic reduction of $4 \mathrm{VP}$ in pyridine containing 30 mmole/1. NaBPh: (top) 8.1 mmole/1. 4VP; (bottom) $15.1 \mathrm{mmole} / 1.4 \mathrm{VP}$.

The cathodic peak current increased with increasing scan rate. Plots of $I_{\mathrm{p}} / \dot{v}^{1 / 2}$ vs $\dot{v}$, where $I_{\mathrm{p}}$ and $\dot{v}$ are the current peak and scan rate, respectively, showed horizontal straight lines (Fig. 3). A $40 \mathrm{mV}$ shift in peak potential was noted for a 5 -fold change in scan rate (from 0.1 to $0.5 \mathrm{~V} / \mathrm{sec}$ ). No linear dependence of cathodic peak current on monomer concentration was observed.

The resistance compensation was not employed because the ohmic drop in the electrolytic cell was negligible $(<0.002 \mathrm{~V})$. The solution of tetraphenylboron in pyridine provided a highly electrical conducting medium. Therefore, the exclusion of the resistance compensation did not affect our experimental results.

\section{Polymerization and Polymer Characteristics}

All polymerizations were carried out under conditions of constant current electrolysis. Because of practical difficulties, polymerization with constant current electrolysis was not performed. 


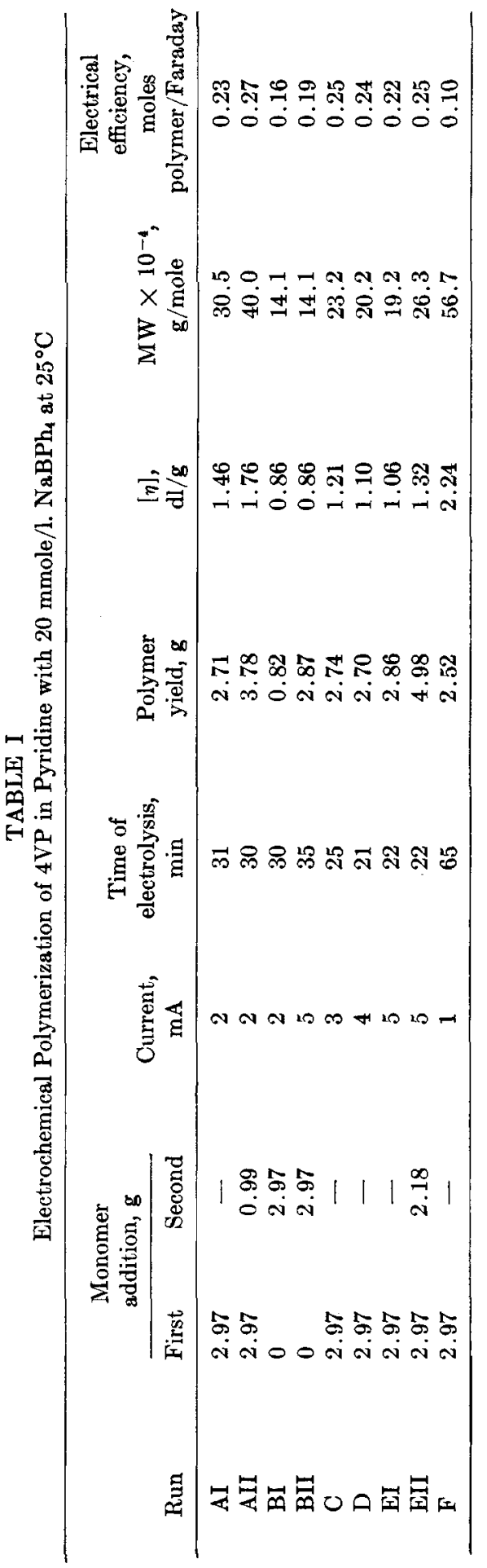




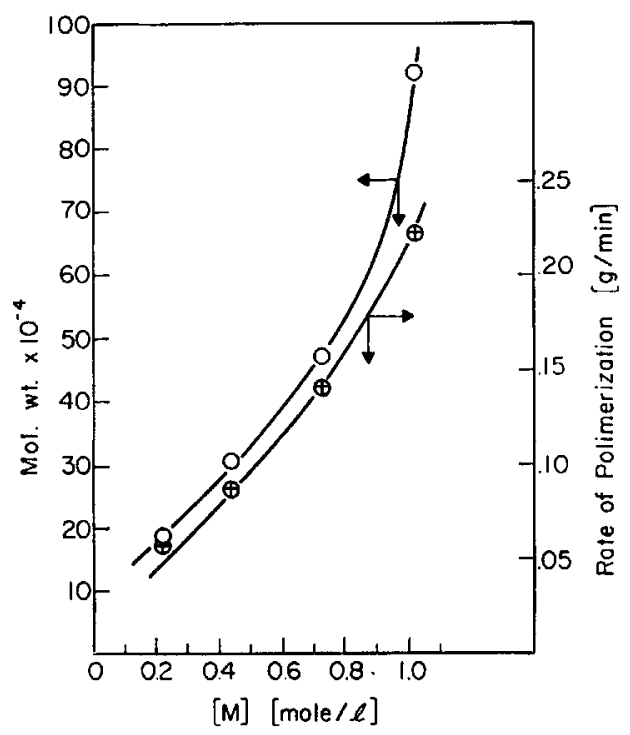

Fig. 4. Polymerization rate $(\Theta)$ and molecular weight $(O)$ vs. monomer concentration for the electrochemical polymerization of $4 \mathrm{VP}$ in pyridine at $25^{\circ} \mathrm{C}$, current density $2 \mathrm{~mA} / \mathrm{cm}^{2}$.

An induction period of $15 \mathrm{~min}$ was observed for polymerization but not for the formation of an orange-red mass around the cathode. Upon passage of the electric current through the 4VP solutions an orange-red mass (characteristic color of living 4VP anions) appeared immediately around the cathode but disappeared quickly in the solution and no polymerization occurred for few minutes. Probably this resulted from living anions formed at the cathode and initially consumed by residual impurities present in the solution. After the impurities had been purged by reaction with the 4VP anions, polymerization started, and the catholyte became viscous and orange-red. The induction period was dependent upon the impressed current.

Upon introduction of air, moisture, or other foreign substances, the catholyte color disappeared. Furthermore the polymerization did not occur without passage of the electric current or if these impurities were originally present. It was found that the polymerized orange-yellow or orange-red solution obtained after cessation of the electrolysis retained the ability to continue the polymerization if additional monomer was supplied to it. In some experiments (AII, EII, Table I), a second ampule of the monomer was introduced into the catholyte compartment after complete polymerization of the original amount. After addition of the second aliquot no current was passed through the solution. The catholyte was stirred for $15 \mathrm{~min}$ and then polymerization was terminated with the addition of a few drops of methanol. The polymer yields were almost equal to the total amounts of monomer used, as shown by comparison with control experiments AI, EI (Table I). The molecular weights of the polymer in- 
creased from 305000 to 400000 (experiments $\mathrm{AI}$ and $\mathrm{AII}$ ) and from 192000 to 263000 (experiments EI and EII).

Integral rates of polymer formation increased, whereas polymer molecular weights decreased with increasing current densities. The observations on polymerization rate and polymer molecular weight are summarized in Table I.

Figure 4 shows the effect of monomer concentration on the integral rate of polymerization and molecular weights at a constant current of $2 \mathrm{~mA}$.

The polymers obtained were white and soluble in $\mathrm{CH}_{3} \mathrm{OH}, \mathrm{CH}_{3} \mathrm{Cl}$, and dimethylformamide, but insoluble in $\mathrm{H}_{2} \mathrm{O},\left(\mathrm{CH}_{3}\right)_{2} \mathrm{CO}$, THF, and petroleum ether. $\mathrm{C}_{6} \mathrm{H}_{6}$ and $\mathrm{C}_{6} \mathrm{H}_{5} \mathrm{CH}_{3}$ were found to swell the polymers. Polymers melted in the range of $160-190^{\circ} \mathrm{C}$. No significant differences were found in the infrared spectra between poly-4VP produced electrochemically in pyridine, in liquid $\mathrm{NH}_{3},{ }^{1}$ or chemically in toluene with $\mathrm{BuLi}^{4}{ }^{4}$

When an equimolar mixture of 4VP and styrene in pyridine was subjected to electrolysis at $2 \mathrm{~mA}$, no copolymer formed, but only poly-4VP, as indicated by the infrared spectra and solubility behavior of the resulting polymers.

\section{Optical Absorption Spectrum}

The absorption spectra of the orange-red solution, obtained by electrolysis of $0.43 \mathrm{~mole} / \mathrm{l}$. $4 \mathrm{VP}$ in pyridine at room temperature, are shown in Figure 5 (curves $a$ and $b$ ). Curve $b$ shows the spectrum measured $20 \mathrm{hr}$ after the measurement of curve $a$. The initial $373 \mathrm{~m} \mu$ peak decreased with time and a distinct peak at $570 \mathrm{~m} \mu$ appeared.

When a solution of $0.3 \mathrm{~mole} / 1.2 \mathrm{VP}$ in pyridine was subjected to electrolysis at $1 \mathrm{~mA}$ for $60 \mathrm{~min}$, the catholyte became viscous and yellow. The

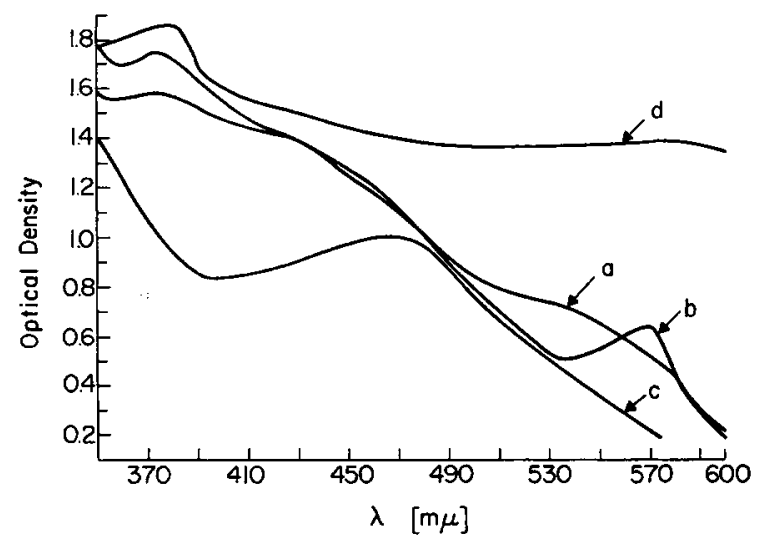

Fig. 5. Absorption spectra of $4 \mathrm{VP}$ and $2 \mathrm{VP}$ in pyridine containing $20 \mathrm{mmole} / \mathrm{l}$. $\mathrm{NaBPh}_{4}$ at $25^{\circ} \mathrm{C}$ : (a) $0.43 \mathrm{~mole} / 1.4 \mathrm{VP}$ electrolyzed for $30 \mathrm{~min}$ at a current of $2 \mathrm{~mA}$; (b) spectrum measured $20 \mathrm{hr}$ after the measurement of curve a; (c) 0.30 mole/l. $2 \mathrm{VP}$ electrolyzed at $1 \mathrm{~mA}$ for $60 \mathrm{~min}$; $(d)$ pyridine containing $20 \mathrm{mmole} / \mathrm{l}$. $\mathrm{NaPBh}_{4} \mathrm{elec-}$ trolyzed in the absence of monomer at $3 \mathrm{~mA}$ for $28 \mathrm{~min}$. 
liquid showed an absorption maximum at $470 \mathrm{~m} \mu$ (curve $c$ in Fig. 5). The color and absorption spectrum of the yellow solution remained unchanged for a long period of time.

The electrolysis of $\mathrm{NaBPh}_{4}$ in pyridine $(3 \mathrm{~mA}$ for $28 \mathrm{~min}$ ) resulted in the formation of deep yellow color at the catholyte. This color gradually changed to green and finally to blue. Curve $d$ in Figure 5 represents the spectrum of the blue solution displaying a peak at $380 \mathrm{~m} \mu$.

To explore whether or not yellow and blue solutions of reduced pyridine would initiate polymerization of 4VP, experiments BI and BII (Table I) were performed. In run BI pyridine was electrolyzed until it became deep yellow and then $4 \mathrm{VP}$ was introduced. The catholyte rapidly became pinkred and subsequently turned again to faint yellow. In experiment BII pyridine was subjected to electrolysis until the catholyte became deep blue. On addition of $4 \mathrm{VP}$, the blue solution again became deep pink-red and viscous. In both cases polymers with high molecular weight were obtained (Table I).

\section{DISCUSSION}

The results presented in the previous section clearly show that $4 \mathrm{VP}$ can be polymerized electrochemically in pyridine containing $\mathrm{NaBPh}_{4}$. As a result of the passage of the electrical current, polymer formation occurred exclusively in the cathode compartment, and the catholyte became yellow or orange-red depending on current intensity while the anolyte remained colorless. In the cyclic voltammetry experiments the peak at $-2.15 \mathrm{~V}$ (Fig. 2) is attributed to direct electron transfer from the Pt cathode to the vinyl bond of $4 \mathrm{VP}$ resulting in the formation of monomeric radical anions. This has been further corroborated by the linear dependence of $I_{\mathrm{p}} / \dot{v}^{1 / 2}$ with (Fig. 3), as is expected for an electrochemical mechanism. ${ }^{6}$ Direct elec-

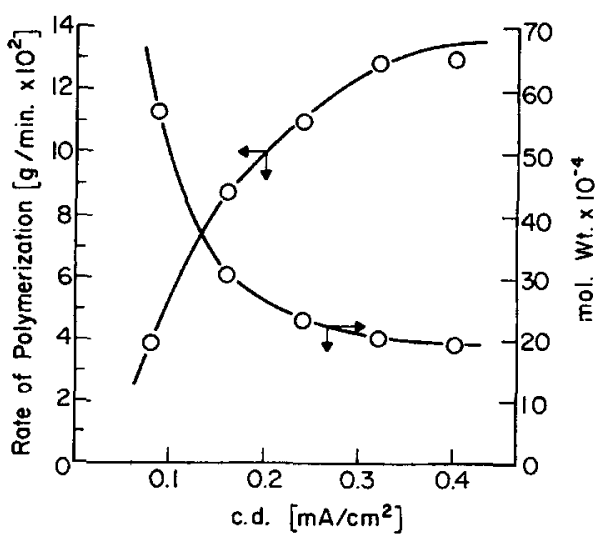

Fig. 6. Polymerization rate and molecular weight vs. current density for the electrochemical polymerization of 0.43 mole/l. $4 \mathrm{VP}$ in pyridine with $20 \mathrm{mmole} / \mathrm{l}$. NaBPh at $25^{\circ} \mathrm{C}$. 
tron transfer to vinyl monomers was previously reported. ${ }^{1,7}$ The absence of the anodic peak, when the scan was limited to $-2.25 \mathrm{~V}$, points out that anodic electron transfer from the radical anions is slow or irreversible or the radical anions react rapidly.

The integral rates of polymer formation increased and polymer molecular weights decreased with increasing current density (Fig. 6). This is expected, since a high current density increases the concentration of initiating species which in turn enhances propagation rate and decreases polymer molecular weight. Polymerization rates and molecular weights increased with monomer concentration (Fig. 4). A fivefold increase in monomer concentration enhanced the molecular weight by about a similar amount. In the $4 \mathrm{VP}$-liquid $\mathrm{NH}_{3}$ systems, ${ }^{1}$ the integral rates of polymerization and the polymer molecular weights were found to be almost independent of monomer concentration. The authors ${ }^{1}$ had suggested that hindrance of monomer diffusion into polymer deposits, in which active centers were imbedded, caused the polymerization rate and molecular weight to be independent of the initial monomer concentration. Unlike liquid $\mathrm{NH}_{3}$, pyridine furnished a homogeneous reaction medium, hence polymer growth took place preponderantly in the liquid phase where monomer molecules were easily accessible to growing polymer chains. Therefore in such systems, where chain transfer and termination processes are absent or at least negligible, the molecular weight of polymer is likely to increase with the increase of monomer concentration. The two-stage monomer addition experiments (AI and AII, and EI and EII, Table I) indicate that the polymerization of $4 \mathrm{VP}$ in pyridine is nonterminating.

The electrolysis of an equimolar mixture of $4 \mathrm{VP}$ and styrene in pyridine resulted exclusively in the formation of poly-4VP. This is to be expected in anionic polymerization, because $4 \mathrm{VP}$, having relatively high electron affinity, is more reactive than styrene with growing polymeric anions. It should be noted that the increase in molecular weight upon the addition of the second aliquot of monomer does not exactly correspond to that expected if all the monomer were employed to add to the existing chains. Secondary reactions may have been responsible for this.

The electronic spectra of living polymer of 2VP and 4VP in THF exhibit an absorption maximum at $315 \mathrm{~m} \mu .^{8}$ In pyridine nonterminated poly-4VP showed a peak at $373 \mathrm{~m} \mu$ (Fig. $5 a$ ) and nonterminated poly-2VP at $450 \mathrm{~m} \mu$ (Fig. 5c). Since pyridine is a more polar solvent than THF (dielectric constants: pyridine-12.3, THF-7.6), carbanions derived from $2 \mathrm{VP}$ and 4 VP may exist in pyridine as free anions or solvent separated ion pairs. Therefore the occurrence of red bathochromic shifts is likely.9.10 It was found that the initial $373 \mathrm{~m} \mu$ peak decreased with time and a new peak at $570 \mathrm{~m} \mu$ appeared (Fig. $5 b$ ). This change may be attributed to side reactions of living polymers. ${ }^{11}$

In previous work ${ }^{12-14}$ it was discovered that the reduction of anhydrous pyridine by alkali metals results in the formation of a yellow solution which gradually changes to green, then blue. 
The electronic spectrum of the yellow solution was found to contain a broad band at $330 \mathrm{~m} \mu$ and that of the blue solution two bands at 381 and $590 \mathrm{~m} \mu$. The yellow color was attributed to pyridyl radical anions and the blue color to 4,4'-bipyridyl radical anions. ${ }^{14}$ The absorption spectra at 381 and $590 \mathrm{~m} \mu$ are equivalent to those in the same region found in this study (Fig. $5 d$ ). The results from runs BI and BII demonstrate that the electrolyzed solutions of pyridine are capable of initiating the polymerization of $4 \mathrm{VP}$ resulting in the formation of high molecular weight polymers. Pyridyl and/or 4,4'-bipyridyl radical anions may be responsible for initiating polymerization.

\section{Mechanism of Polymerization}

In the present system three possible modes of initiation can be visualized, namely: (1) direct electron transfer from cathode to monomer:

$$
\mathrm{M}+e^{-} \rightleftharpoons \mathrm{M}^{-}
$$

where $M, e^{-}$represent monomer and electron, respectively: (2) electron transfer to monomer from $\mathrm{Na}$ metal deposited on the cathode:

$$
\begin{gathered}
\mathrm{Na}^{+}+e^{-} \rightleftharpoons \mathrm{Na} \\
\mathrm{Na}+\mathrm{M} \rightleftharpoons \mathrm{M}^{-} \mathrm{Na}^{+}
\end{gathered}
$$

(3) initiation by pyridyl radical anions formed by reduction of pyridine:<smiles>CCC[Se-]c1ccccc1</smiles><smiles>[C+]C=CCC=CCC</smiles>

$\mathrm{N}_{\odot}$<smiles>C1=CCCCC1</smiles>

$\mathrm{N}_{\odot}$<smiles>C[C@@H]1C=CC=CC1</smiles>

$\mathrm{N}_{\ominus}$<smiles>c1ccccc1</smiles>

$\mathrm{N}_{\odot}$

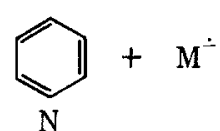

$\mathrm{N}$

The results from cyclic voltammetry show that the reduction potential of the solvent (or $\mathrm{NaBPh}_{4}$ ) is more negative than that of 4VP (Fig. 2). Therefore, scheme (1) would be preponderant for initiation, although other initiation processes cannot be ruled out because of close proximity of reduction potentials of monomer, solvent, and salt. Ideal anion-radical growth is characterized by an electrochemical efficiency of 1 while for ideal dianion growth it is 0.5. The electrochemical efficiency is given by the average number of polymer molecules produced per electron. The observed electrochemical efficiency is about 0.22 (Table $\mathrm{I}$ ). This is attributed to residual impurities present in the polymerizing solution, and it is supported by the observation of an induction period during polymerization. It is probable that radical anions formed at the electrode dimerize, resulting in the formation of dianions which lead dianionic growth of polymerization. The sensitivity of the reaction to air, moisture, and protic substances, the high polymerization rate, and the nonterminating behavior suggest that anionic intermediates are responsible for the propagation process. 
In the liquid $\mathrm{NH}_{3}-4 \mathrm{VP}$ system, the propagation step occurred near the electrode surface or within the polymer deposited on the cathode because the resulting polymers were insoluble in liquid $\mathrm{NH}_{3}$ and adhered to the electrode. In contrast to this behavior, polymer formation in pyridine occurred at the electrode and in solution because of the solubility of the growing polymers.

\section{CONCLUSIONS}

The experimental findings of this work revealed the following.

(1) The passage of an electric current through 4VP solutions of pyridine in the presence of dissolved $\mathrm{NaBPh}_{4}$ produces homogeneous, nonterminating polymerizations of the monomer at smooth Pt cathodes.

(2) Poly-4VP of high molecular weight $\left(\sim 10^{5}\right)$ is formed in the catholyte.

(3) The reduction potential of the monomer at $\mathrm{Pt}$ cathode $(-2.2 \mathrm{~V})$ is more positive than that of pyridine and $\mathrm{NaBPh}_{4}(>-2.48 \mathrm{~V})$.

(4) The polymerization rate and polymer molecular weight increased with monomer concentration. The rate of polymerization decreases while the polymer molecular weight increases with current density.

(5) After having been subjected to electrolysis, pyridine initiates polymerization without further passage of electrical current and produces polymer in high yield and with high molecular weight.

We wish to acknowledge with thanks fruitful discussions with Dr. D. G. Laurin.

The present work was made possible through financial support from the National Science Foundation and the Dreyfus Foundation. This support is gratefully acknowledged.

\section{References}

1. D. G. Laurin and G. Parravano, in Macromolecular Chemistry Brussels-Louvain 1967 (J. Polym. Sci. C, 22), G. Smets, Ed., Interscience, New York, 1968, p. 103.

2. S. Tazuke and S. Okamura, J. Polym. Sci. A-1, 4, 141 (1966).

3. J. Y. Yang, W. E. McEwen, and J. Kleinberg, J. Amer. Chem. Soc., 79, 5833 (1957).

4. P. P. Spiegelman and G. Parravano, J. Polym. Sci. A, 2, 2245 (1964).

5. D. J. G. Ives and G. J. Janz, Eds., Reference Electrodes Theory and Practice, Academic Press, New York, 1961, p. 203.

6. R. S. Nicholson and I. Shain, Anal. Chem., 36, 706 (1964); ibid., 37, 178 (1965).

7. B. L. Funt and F. D. Williams, J. Polym. Sei. A, 2, 865 (1964).

8. C. L. Lee, J. Smid, and M. Szware, Trans. Faraday Soc., 59, 1192 (1963).

9. R. Waack and M. A. Doran, J. Phys. Chem., 67, 148 (1963).

10. T. E. Hogen-Esch and J. Smid, J. A mer. Chem. Soc., 88, 307 (1966).

11. G. Spach, M. Levy, and M. Szwarc, J. Chem. Soc., 1962, 355.

12. A. Carrington and J. Santos-Veiga, Mol. Phys., 5, 21 (1962).

13. R. L. Ward, J.Amer. Chem. Soc., 83, 3623 (1961).

14. C. D. Schmulbach, C. C. Hinckbly, and D. Wasmund, J. Amer. Chem. Soc., 90, 6600 (1968).

Received April 11, 1969

Revised July 11, 1969 